\title{
OPPORTUNITIES FOR USING AGENT-BASED SOCIAL SIMULATION AND FUZZY LOGIC TO IMPROVE THE UNDERSTANDING OF DIGITAL MENTAL HEALTHCARE SCENARIOS
}

\section{Owen Barnes}

University of Nottingham

School of Computer Science

Nottingham, NG8 1BB

owenebarnes@gmail.com
Dr Peer-Olaf Siebers

University of Nottingham

School of Computer Science

Nottingham, NG8 1BB

peer-olaf.siebers@nottingham.ac.uk

\begin{abstract}
Agent-Based Social Simulation (ABSS) has seen success in studying emerging behaviour in social contexts. In this paper, we explore the use of ABSS to improve the understanding of digital mental healthcare scenarios. Our exploratory study focuses on understanding how different age generations within a population react to the implementation of digital mental health therapies. Our model uses a complex social media network where agents can communicate and alter their opinions over time. In this paper we also present some technical novelty. We introduce interval type-2 fuzzy logic as an option for modelling human decision-making and accounting for the uncertainty that exists when modelling complex systems. Fuzzy Logic is a concept particularly well suited to formalise and deal with imprecise concepts. The work presented here seeks to encourage Social Simulation modellers to experiment more with interval type- 2 fuzzy logic.
\end{abstract}

Keywords: Agent-Based Modelling, Social Simulation, Fuzzy Logic, Digital Mental Health, Trust

\section{INTRODUCTION}

With the recent emergence of data-driven technologies, the popularity of digital mental healthcare has risen significantly (Arigo et al. 2019). With this comes the opportunity to use simulation to further our understanding of how digital mental healthcare may affect the population when implemented. Since this area is recent to the past decade, there is little insight into how we can advance our abilities to model such technologies, especially when considering the social and ethical implications of the technology. In this paper we consider the following possible future digital mental healthcare scenario: Therapy sessions are inspired by chatbot therapy, which are one-to-one therapies taking place between digital chatbot therapists and patients (Health Europa 2019). After weekly sessions, the trust value and the psychological condition of patients change, and trust levels can also be altered through access to social media. Over time, some trends should be observable that indicate the impact of healthcare quality and social communication on the trust of the population as a whole, and on the trust of specific groups within the population in particular. Our study considers different generations of people (from Millennials to Baby Boomers), and explores how different groups of people responded to different scenarios, for example when the quality of the provided therapy is poor.

The goal of this paper is twofold. On the one hand, we are interested to investigate how AgentBased Social Simulation (ABSS) can be used in the context of studying digital mental healthcare. For this purpose, we use the Engineering ABSS (EABSS) framework by Siebers and Klügl (2017). This framework supports the conceptualisation of ABSS models, using the concept of co-creation, as well as software engineering tools and methods. It drives the model development process and documents the outcome of this process. It is used to provide transparency to viewers and to improve the ability for multi-disciplinary stakeholders to understand the model without technical jargon. On the other hand, we want to look at novel ways of representing decision-making processes in agents. For this we 
test whether interval type-2 fuzzy logic could provide modellers with a realistic decision-making representation when modelling these types of scenarios. Interval type- 2 fuzzy logic benefits from being able to handle uncertainty with promising results, but has seen little application within ABSS.

In the remainder of this paper we first provide background information on ABSS, fuzzy logic and its use in ABSS, and digital mental healthcare (Section 2). We then present a detailed description of our conceptual model for simulating digital mental healthcare scenarios, using the EABSS framework as a documentation tool (Section 3). Next, we briefly describe the tools we used for the implementation of our conceptual model (Section 4). We then present our experimental results and findings with regards to our established hypotheses, and comment on interesting insights (Section 5). Finally, we provide a summary of achievements, limitations, and how to overcome these limitations in the future (Section 6).

\section{BACKGROUND}

\subsection{Agent-Based Modelling and Social Simulation}

In Agent-Based Modelling (ABM) a system is modelled as a collection of autonomous decisionmaking entities (agents) where each agent individually assesses its situation and makes decisions on the basis of a set of rules (Bonabeau 2002). Individual agents interact with each other and their environment to produce complex collective behaviour patterns at system level. Agents are designed to mimic the behaviour of their real-world counterparts; they are capable of making autonomous decisions and showing proactive behaviour. Agent-Based Simulation (ABS) is a powerful simulation paradigm that can be used for conducting what-if analysis of human centric systems (Siebers and Aickelin 2008). By developing models of complex social systems and studying their evolution through simulated time, researchers have an artificial lab where they can observe the interactions between social agents and processes and their consequences. Such artificial labs can be used for small-scale exploratory studies as well as large-scale decision support applications. ABS is a bottomup approach and is used in situations for which individual variability between the agents cannot be neglected. It allows understanding how the dynamics of many real systems arise from traits of individuals and their environment. It allows modelling a heterogeneous population where each agent might have personal motivations and incentives, and to represent groups and group interactions. Social Simulation (SS) studies socio-economic phenomena by investigating the social macrostructures and observable regularities generated by the behaviour and relationships between individual social agents, and the environment in which they act. This is useful for policy decision support in many scenarios, including transport, housing, education, or healthcare. ABSS is a variation of ABS and SS, which looks at modelling social behaviour using agent technologies; it is commonly described as a multidisciplinary intersection between agent-based computing, social sciences, and computer simulation (Davidsson 2002).

\subsection{Fuzzy Logic in the Context of Social Simulation}

Fuzzy Logic is defined by Zadeh (1988) as the logic underlying approximate, rather than exact modes of reasoning, considering "degrees of truth" rather than the usual "true or false" Boolean logic. It is a concept particularly well suited to formalise and deal with imprecise concepts (Izquierdo et al 2015). When using fuzzy logic in simulation contexts, the most popular use of fuzzy logic is by using inference systems. Fuzzy inference systems are able to provide modellers with an ability to model the subjective uncertainties which arise in ABSS, such as when modelling social relationships and exchanges between agents (Raoufi and Rayek 2015). By providing the system with inputs, the system can produce an outcome based on the modelled scenario. Type-1 fuzzy inference systems model uncertainty with the use of membership functions which correspond to the value an input may have to a specific set. Interval type-2 fuzzy systems go a step further by also making the membership functions a fuzzy set. In a type-1 membership function, the set modelled must be precisely defined by the modeller, while in interval type- 2 membership functions, the modeller can be vague in their definition. The modeller defines an upper and lower membership function which forms an area called the footprint of uncertainty. Within the footprint of uncertainty, an embedded set is produced which is 
the equivalent of a type-1 set. This is useful when trying to model subjective concepts such as emotion, which is not the same for every agent. When modelling these concepts, the larger area of the footprint of uncertainty means more uncertainty is represented in the definition of the membership function. For a more in-depth coverage of this topic please refer to Mendel et al. (2006). The applications of type-1 fuzzy logic to ABSS are extensive, but we have only found one application of interval type-2 fuzzy logic to ABSS (Castañón-Puga et al. 2014) and none documented with regards to healthcare.

\subsection{Digital Mental Health and Healthcare in the Context of Social Simulation}

The rise of big data within the world means that quantitatively driven solutions can be applied to a range of problems. Examples of these applications include devices which track your health statistics by counting the amount of steps you make in a day (Kerner and Goodyear 2017), using motion tracking to analyse your sleep performance (Hamida et al. 2015), or recently, offering a way for users to communicate their emotions and feelings to act as a digital therapeutic bot (Fitzpatrick et al. 2017). Since popularity has grown, there have naturally been efforts to implement this sort of digital technology to more specialised areas of healthcare. This consists of developing AI in ways which can help provide alternative methods of treating mental health issues successfully such as depression, anxiety, and dementia (Fernandez-Sotos et al. 2019). We found very few papers during our literature review which explored digital mental health scenarios, and it was found that those papers were focused on understanding the social and ethical implications of digital mental health rather than looking at the service provision aspects. As far as we could see, the simulation of digital therapies appears novel and untouched by the simulation modelling community.

\section{CONCEPTUAL MODEL}

\subsection{Approach}

The EABSS framework provides the core of a methodology which supports ABSS model development and documentation in a structured way. Full details about the framework and its application can be found in Siebers and Klügl (2017). The EABSS framework is grounded in the concept of co-creation (Mitleton-Kelly 2003) and ideas from software engineering (Sommerville 2015), but it can also be used by individuals. In the latter case the individual needs to consider the perspective of stakeholders (i.e. slip into their roles) during each process step. The framework consists of an Analysis and a Design part, as depicted in Figure 1. For capturing different types of information, it uses the Unified Modelling Language (UML) notation (Fowler 2004) extensively.

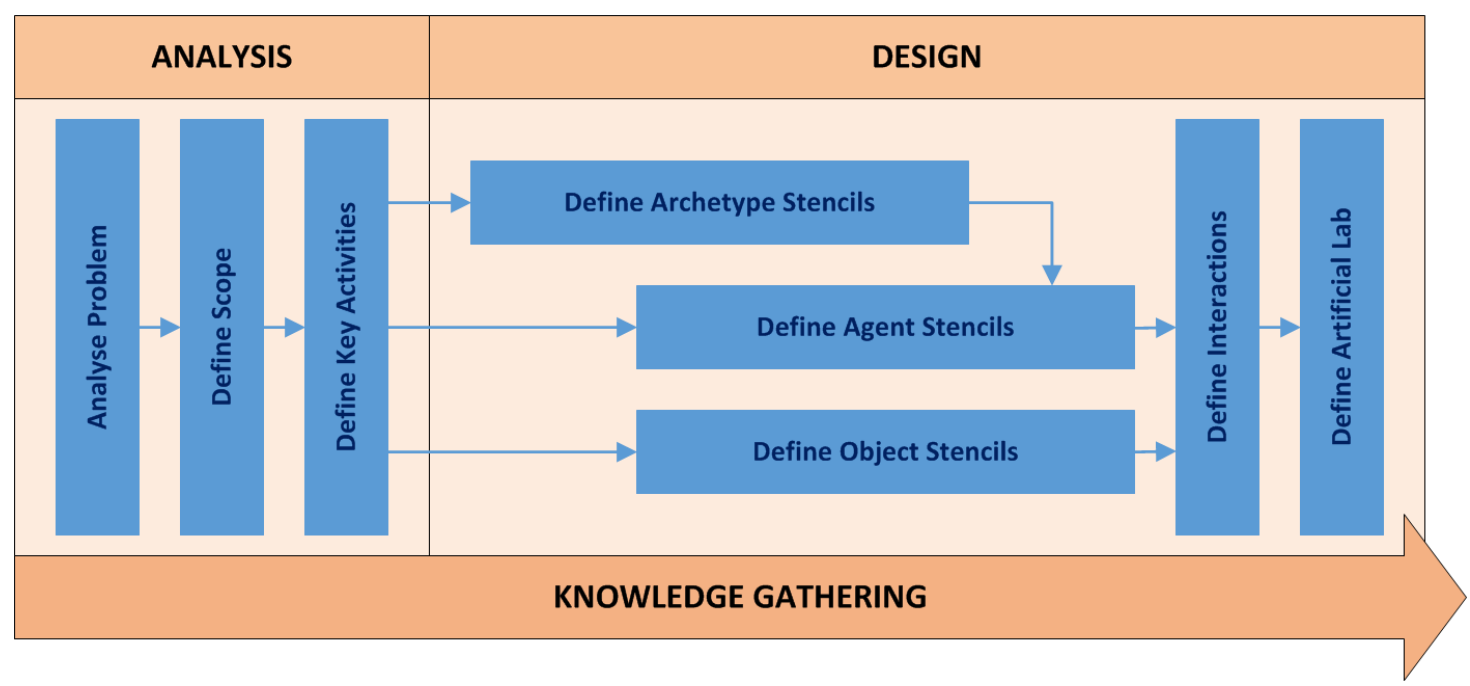

Figure 1 A high-level overview of the EABSS (after Siebers and Klügl 2017) 


\section{Barnes and Siebers}

\subsection{Analysis}

\section{Analysing the Problem}

Experience with digital therapy is still in its infancy. The aim of our study is to better understand how people react towards digital therapy, with people modelled based on generation groups: Millennials, Generation-X, and Baby Boomers. In this paper we focus on testing two hypotheses: (1) "The more access to social interaction agents have, the more sensitive the simulation would be when certain parameters (e.g. trust) are altered" and (2) "Competence of digital therapies (i.e. our 'Session' in the simulation) will play a large role in defining the output trust of the population".

Experimental factors are model parameters that can be altered to test our hypotheses. For our purpose the experimental factors consist of (1) the proportion of different generations of agents, (2) the initial level of trust of the population tested, (3) the competence of digital therapies, and (4) the level of access agents have to the social network. By altering these model parameters, it is possible to investigate the effect they have on the dynamics of the system over time.

Responses are the outputs from the model which enable us to test our hypotheses. ABSS allows to collect micro and macro-level data in form of time series and averages over time. The trust variable was the primary response for testing our hypotheses; at the macro-level this captured the aggregated trust of the population, and at the micro-level it looked at individual agent's trust level evolution during the simulation.

\section{Scope}

This stage involves defining the level of abstraction appropriate to test the hypotheses, identifying relevant actors, relevant elements of the physical environment, as well as social and psychological aspects that might be relevant for the modelling. The agreed scope is presented in Table 1 .

Table 1 Resulting scope table

\begin{tabular}{|c|c|c|c|}
\hline Category & Element & Decision & Justification \\
\hline \multirow[b]{2}{*}{ Actor } & Person (Patient) & Include & Subject which is being observed \\
\hline & Session & Include & A virtual 'therapist' providing services to patients \\
\hline \multirow{4}{*}{$\begin{array}{l}\text { Physical } \\
\text { Environment }\end{array}$} & Hospital & Exclude & \multirow{3}{*}{$\begin{array}{l}\text { No benefits of representing these elements within the } \\
\text { environment as physical entities }\end{array}$} \\
\hline & Home & Exclude & \\
\hline & Therapy & Exclude & \\
\hline & Spatial node & Include & $\begin{array}{l}\text { Used to measure distance between agents and defining the } \\
\text { location of agents during the simulation }\end{array}$ \\
\hline \multirow{8}{*}{$\begin{array}{l}\text { Social and } \\
\text { Psych. Aspects }\end{array}$} & Person social influence & Include & Used to help determine the outcomes of social interactions \\
\hline & Person condition & Include & $\begin{array}{l}\text { Tracks the outcome of session treatment, and is what makes } \\
\text { people admit themselves to care }\end{array}$ \\
\hline & Person trust & Include & Our primary response (and therefore fundamental to the model) \\
\hline & Person age & Include & Important for determining social media usage \\
\hline & Desire & Exclude & \multirow{3}{*}{$\begin{array}{l}\text { Psychological theory was initially considered, but later not used } \\
\text { due to time constraints }\end{array}$} \\
\hline & Volatility & Exclude & \\
\hline & Arousal & Exclude & \\
\hline & Stereotype & Exclude & Captured by person age \\
\hline \multirow[t]{2}{*}{ Other } & Social network & Include & $\begin{array}{l}\text { Agents can interact with each other, in a realistic manner through } \\
\text { using friendLink networks to send messages }\end{array}$ \\
\hline & Therapy system & Include & System boundary used by people who require digital therapy \\
\hline
\end{tabular}

\section{Defining Key Activities}

This stage involves linking actors to use cases (which represent the key activities). Figure 2 defines how actors can interact. Bubbles represent relevant use cases. Associations between actor and use case indicate which actor is involved in which use case. Relationships between two use cases specify common functionality and simplify use case flows. 


\section{Barnes and Siebers}

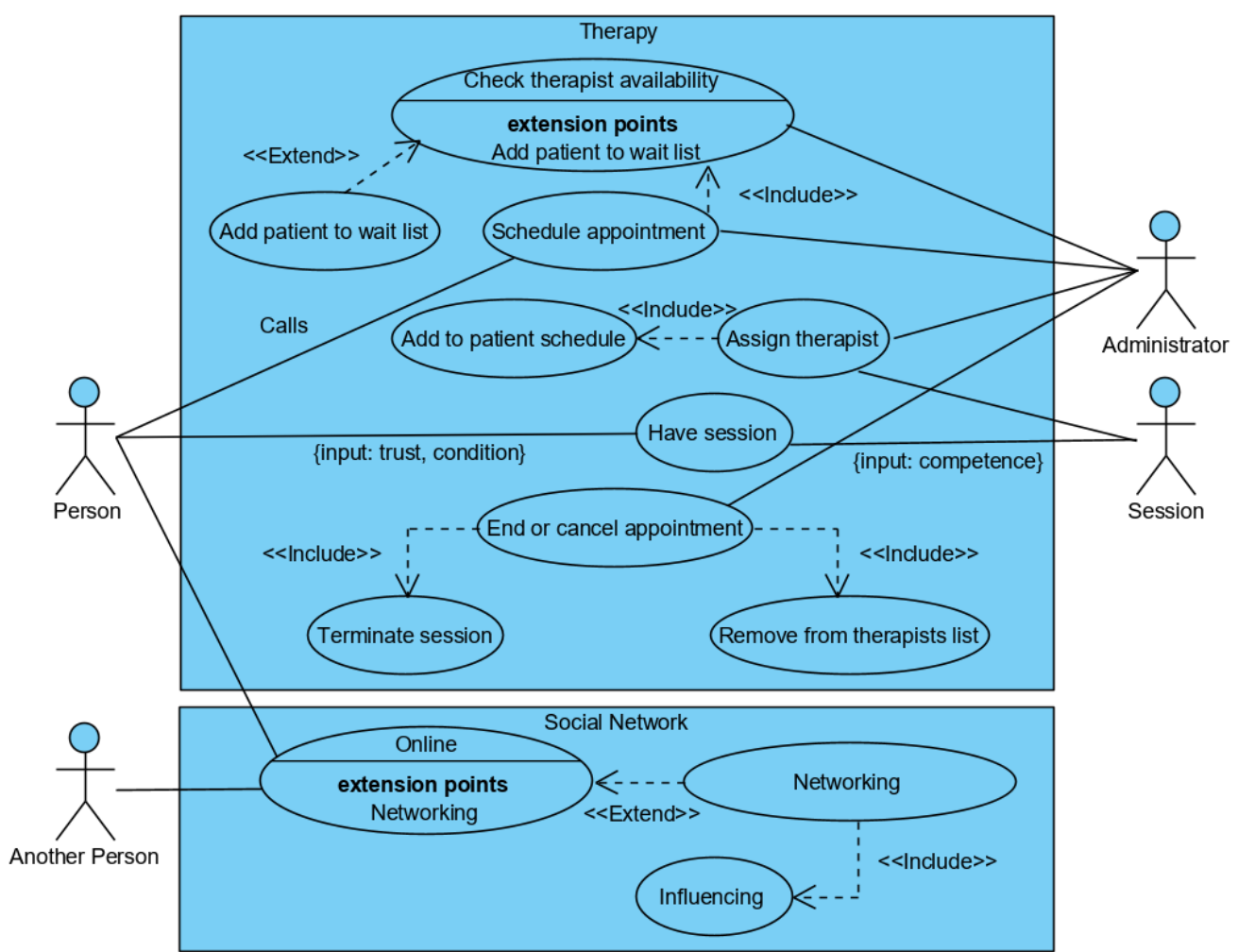

Figure 2 Resulting use case diagram

\subsection{Design}

\section{Archetypes}

This stage involves defining archetypes, based on what we found in the literature. We distinguish three generations, based on age range. From the eMarketer (2019) stats we know how many people within a specific age range use social media, and from Globalwebindex (2018) we know roughly how much time people spend online each day, using social media. A summary of the data can be found in Table 2. We use this information as a stochastic element of the model that resembles the likelihood of an agent using social media at any point during the day.

Table 2 Resulting archetype definitions

\begin{tabular}{|l|c|c|c|}
\hline \multicolumn{1}{|c|}{ Name } & Age range & $\begin{array}{c}\text { Social media users } \\
\text { [\% of age range } \\
\text { members] }\end{array}$ & $\begin{array}{c}\text { Use of social media } \\
\text { [hours per day] }\end{array}$ \\
\hline Millennials & $1980-1994$ & 90.4 & 2.38 \\
\hline GenerationX & $1965-1979$ & 77.5 & 1.49 \\
\hline Baby Boomers & $1944-1964$ & 48.2 & 1.12 \\
\hline
\end{tabular}

\section{Agents}

This stage involves defining the states that entities can be in and the dependencies between these states in form of transitions. UML state machine diagrams (statecharts) are used to present this information. Figure 3 contains the statecharts for all agents identified as actors in Table 1. There are two statecharts for capturing a Person agent's states. The first tracks the possible condition of a person. People in the 'stableCondition' state (holding a condition value of 50+) do not require digital therapy. If their condition value drops below 50, they will arrange a digital therapy session (via selfadmission) with the administrator. The second statechart controls a Person agent's social media usage, where the rate at which a person goes online is defined by their archetype. The Admin and Session agents are secondary agents that provide services to the Person agent. When Person agents get into an unstable condition, they request a session by contacting the Admin agent. The Admin agent then 


\section{Barnes and Siebers}

creates a session in form of an abstract agent that represents a virtual therapist providing digital therapy sessions.

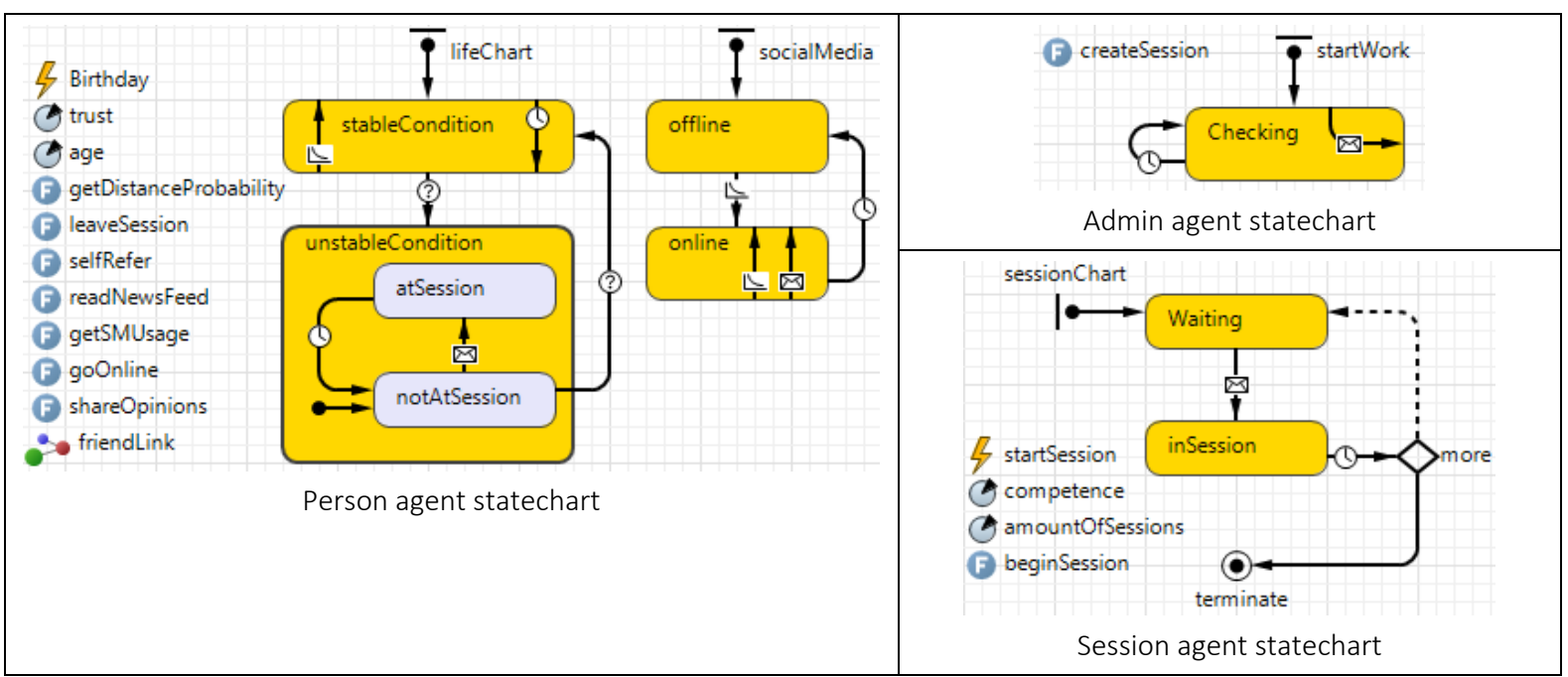

Figure 3 Statecharts of the actors identified in the scope table

\section{Objects}

The only objects we consider in relation to the environment for our model are spatial nodes. They allow us to measure the distance between Person agents, and to graphically represent their location and communication links within a defined space.

\section{Interactions}

The interaction between different agent types is described in Section 3.3, Subsection "Agents". Here we focus on the interaction (online communication and its effects in relation to opinion dynamics) between Person agents (friend links). We model the social media network using an algorithm that connects agents depending on their proximity and social similarity. This algorithm produces a probability that is applied once agents go online, providing realistic communication and influencing effects. More information about the algorithm can be found in Goldenberg \& Levy (2009).

\section{Artificial Lab}

The artificial lab is responsible for providing methods to collect aggregated outputs of the model or provide global methods that can be accessed by all agents. Notable elements of our artificial lab are the "social feed", which emulates a social media newsfeed, and some debugging methods to ensure input parameters to fuzzy inference systems are within bounds.

\section{IMPLEMENTATION}

For the implementation of the conceptual model described in Section 3, we used AnyLogic 8.5 (https://www.anylogic.com/). This is a multi-paradigm simulation IDE that supports (amongst others) the ABM paradigm. It is (relatively) easy to use, yet not restrictive, as it includes a high-level graphical modelling language and allows users to extend the model with custom low-level Java code.

For the implementation of opinion dynamics (covering opinions of agents when communicating across the social media channel) and outcomes of digital therapy sessions, we used fuzzy logic, to see how it accounts for the uncertainty that exists within such systems. We embedded the fuzzy logic as follows. The first fuzzy logic system represents how somebody ( $\mathrm{p} 1)$ online may react to another person's (p2) opinion of digital therapies, which is modelled with an attribute 'Trust'. For agent $\mathrm{p} 1$ the system takes three inputs, the trust of agents $\mathrm{p} 1$ and $\mathrm{p} 2$, and the 'social influence' variable of $\mathrm{p} 1$. The system returns a value between $[-0.05,0.05]$ which is added to $\mathrm{p} 1$ 's trust variable. The second fuzzy logic system provides an output to reflect the reaction of a person to the digital therapy, adjusting $\mathrm{p} 1$ 's 'Trust' and 'Condition' attribute. The system takes three inputs, involving the competence of the session agent, p1's Trust, and p1's Condition. If the person has a positive experience, their values 


\section{Barnes and Siebers}

increase, which is then reflected in future communication on social media. This produces a 'word-ofmouth' social network that offers realistic communication within the model. For fuzzy logic support within AnyLogic, we embedded the Juzzy library (Wagner 2013). It is a Java based toolkit for type-1, interval type-2 and general type- 2 fuzzy logic and fuzzy logic systems. The library provides modellers with a clear and well thought through syntax which can be implemented into AnyLogic by using an external class file. It offers a user-friendly interface for less technical stakeholders, to define the fuzzy logic elements.

Our model is available for download at https://www.comses.net/. Our model has been verified with the help of visual debugging support and several external modellers. In order to ensure results accurately reflect the mean performance of our stochastic simulation model, we use the confidence interval method $(\alpha=5)$ to find the required number of replications. The test indicated that four replications of every iteration were required when collecting results. We also conducted a comparison test between fuzzy and crisp decision-making using a method proposed by $\mathrm{Vu}$ et al. (2013). The details of our comparison are omitted here due to space constraints but can be found in Barnes (2019). In general, we found that results with the fuzzy implementation corresponds better to what we expect to see in the real world, effectively modelling the hesitancy expected within the first few months of deploying the digital therapy to the population.

\section{EXPERIMENTATION}

We tested two hypotheses as defined in the conceptual model, one dealing with "social interactions" and one dealing with "competence". In this section we define on the hypotheses we are testing, provide the experimental setups and results of our experiments and discuss the findings.

\section{$5.1 \quad$ Testing Hypothesis 1: Social Interactions}

Here we explore the effect that age plays in trust dynamics and how sensitive the output of the model is for each generation (from Millennials to Baby Boomers) when we altered the initial trust level of agents. Hypothesis: "The more access to social interaction agents have, the more sensitive the simulation would be when certain parameters (e.g. trust) being altered". Experimental setup: In order to test this hypothesis, we run the simulation for seven iterations for each generation, changing the initial trust value from 47 to 53 . We then collected our data, and tracked statistics such as the variability between runs, to understand whether we should accept or reject our hypothesis. Results: We found that Millennial and Generation-X runs (who experienced more exposure to the social network) proved sensitive when the initial trust level was altered. Both showed high variance between runs, where the variance between Millennial runs was 113.15, and for Generation-X 111.25; significantly lower than when testing our older generation at 4.827 . The high variance shown on these runs tells us that more access to social media is more likely to affect the final trust value of the population at the end of the simulation. Furthermore, we identified a macro-level trend which represented the hesitancy of the population during early stages of the simulation. From this experiment we learnt that slight changes to initial conditions can play a huge role in the success of implementing digital therapies; and this information could prove useful to stakeholders. Figure 4 shows us that early stages of the simulation are crucial in determining the outcome trust levels of the population, and few situations arise where population established trust once the average trust is below 47. We also found that agents tended to benefit more from using digital therapies when the trust level of the population was higher, because they possessed higher input values which provided a better outcome after every session. We accepted our hypothesis due to the high variance between runs, which increased as agents had more access to the social network. 


\section{Barnes and Siebers}

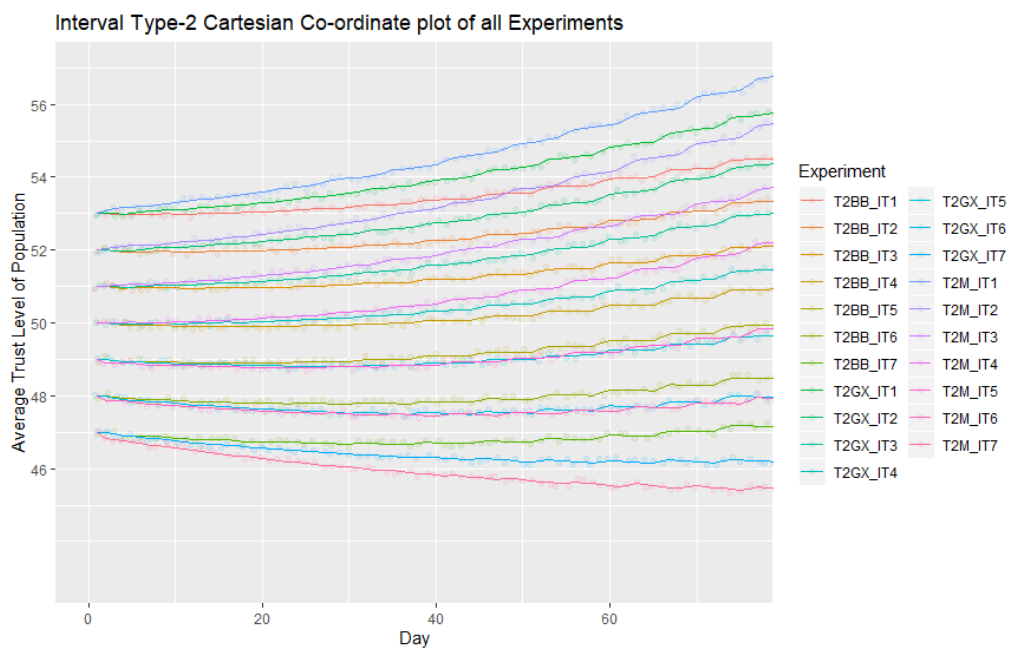

Figure 4 A Cartesian time series plot showing the initial hesitancy of our population across all runs

\subsection{Testing Hypothesis 2: Competence}

Here we test how the simulation reacts when we alter the competence of sessions, which controls the performance of chatbot therapies in the simulation. The output aggregated trust level of the population is expected to alter a lot. We check whether any extremist behaviour emerges, e.g. if the change in competence of our digital therapies cause a significant difference in the overall trust level. Hypothesis: "Competence of digital therapies (i.e. our 'Session' in the simulation) will play a large role in defining the output trust of the population". Experimental setup: To test this hypothesis, we ran scenarios that saw the competence variable of sessions increment from 20 to 80 , in steps of 5 . We tested this to explore whether the variable played a statistically significant impact in the output trust levels of the population, which would lead us to accept our hypothesis. We should expect to see our variance increasing as competence is increased, and for trust to increase consummate to the increase in competence of digital therapies. Results: We found that there was very little difference across all runs, even when comparing extreme scenarios where digital therapies had poor competence, against when they were highly skilled. We can see from Figure 5 that the variance is inconsistent with Generation-X runs.

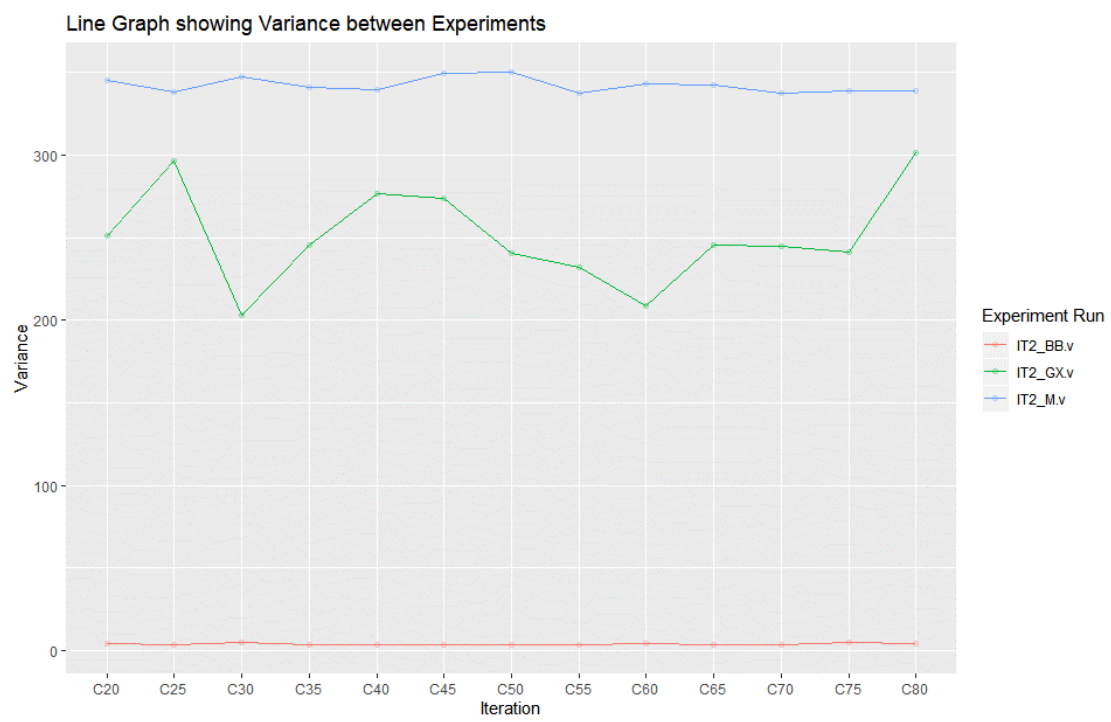

Figure 5 Variance of output trust levels of all runs

Further exploration into this tells us that the attitude of agents, presented in Figure 6, changed the most between day 400 to 600 of the simulation. Figure 6 also shows that there is a wide spread of results when looking at trust level over time, but that all runs establish high trust levels, regardless of 


\section{Barnes and Siebers}

the competence of digital therapies. This was unexpected; we expected to see a lower trust level when the competence of digital therapies is low. We rejected our hypothesis, since the output trust level of the population stayed consistently high regardless of whether our digital therapies were more skilled.

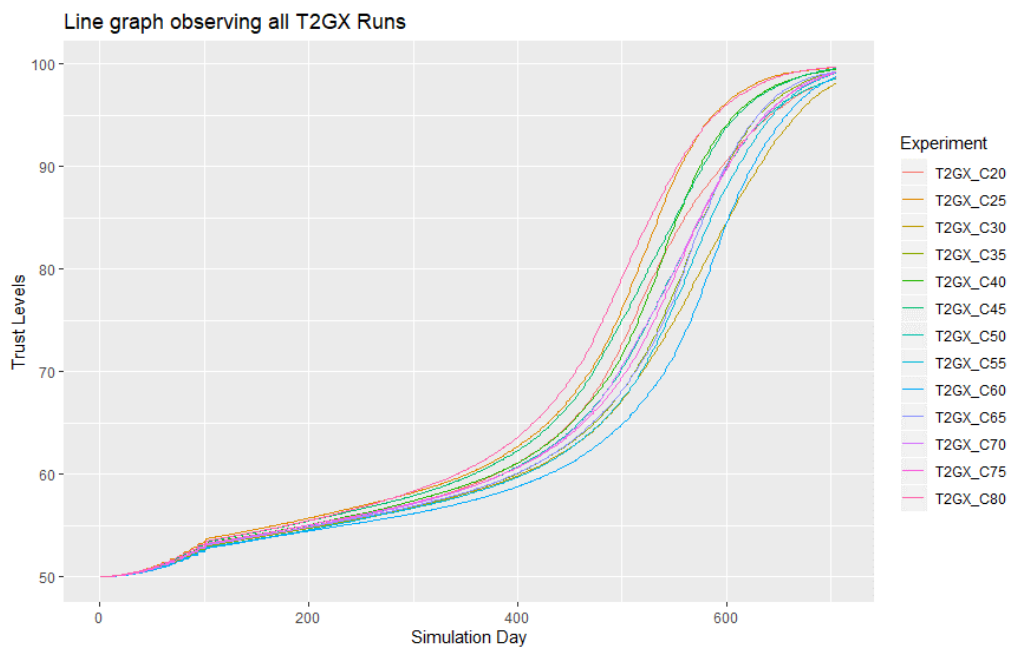

Figure 6 Time series plot of all simulation runs with agents belonging to Generation-X

\section{CONCLUSIONS}

In this paper we have created an exploratory model which introduces a way of simulating the reaction of a diverse population to different digital mental healthcare scenarios. We also showed how interval type-2 fuzzy logic can be successfully implemented to provide decision-making capabilities of agents in uncertain environments. We found patterns in both, micro and macro-level data, which provide useful insight into agents' reactions to digital therapies. This knowledge can be useful for stakeholders when considering the social implications of using digital technologies. The model was unable to be validated and was therefore classified as exploratory. This was perhaps the biggest limitation. Since this is a novel application, the extent to which one can validate the model is limited, and there were no datasets which could be used to compare with the output of the model. In the future we aim to validate the base model, which could be done by organising a workshop with relevant experts and stakeholders.

In conclusion, our project has shown the potential of simulation to investigate scenarios of digital mental health dynamics and the impact appropriate healthcare can have. We hope that in the future more resources are made available to continue the work on this novel research topic.

\section{REFERENCES}

Arigo D, Jake-Schoffman D E, Wolin K, Beckjord E, Hekler E B and Pagoto S L (2019). The history and future of digital health in the field of behavioral medicine. Journal of Behavioral Medicine 42(1): $67-83$.

Barnes O (2019) Exploring the effects of using interval type-2 fuzzy logic with social simulation to help improve the understanding of digital mental health. Dissertation. University of Nottingham.

Bonabeau E (2002). Agent-based modeling: Methods and techniques for simulating human systems. Proceedings of the National Academy of Sciences of the United States of America. 99: Suppl 3.

Castañón-Puga M, Flores-Parra J M, Gaxiola-Pacheco C, Palafox-Maestre L E, Ahumada-Tello E (2014) Extending NetLogo with interval type-2 fuzzy inference systems. In: Second World Conference on Complex Systems (WCCS) 10-13 Nov, Agadir, Morocco.

Davidsson P (2002). Agent based social simulation: A computer science view. Journal of Artificial Societies and Social Simulation, 5(1):7.

eMarketer (2019) US Social Media Users, by Generation, https://www.emarketer.com/chart/226029/ us-social-media-users-by-generation-2019-of-population [Last accessed 01/02/2020]

Fernandez-Sotos P, Fernandez-Caballero A, Gonzalez P, Aparicio A I, Martnez-Gras I, Torio I, Dompablo M, Garca-Fernandez L, Santos J L and Rodriguez-Jimenez R (2019) Digital 
technology for internet access by patients with early-stage schizophrenia in Spain: Multicenter research study. Journal of Medical Internet Research 21(4).

Fitzpatrick KK, Darcy A and Vierhile M (2017) Delivering cognitive behavior therapy to young adults with symptoms of depression and anxiety using a fully automated conversational agent (Woebot): A randomized controlled trial. JMIR Mental Health 4(2).

Fowler M (2003) UML Distilled: A Brief Guide to the Standard Object Modeling Language (Object Technology Series). 3e. Addison-Wesley Professional.

Globalwebindex (2018) Social media across generations (Preview) https://www.globalwebindex.com/ reports/social-media-across-generations [Last accessed 01/02/2020]

Goldenberg J and Levy M (2009) Distance is not dead: Social interaction and geographical distance in the Internet era. arXiv:0906.3202.

Hamida, S T B , Ahmed B, Cvetkovic D, Jovanov E, Kennedy G and Penzel T (2015). A new era in sleep monitoring: The application of mobile technologies in insomnia diagnosis. In: Mobile Health. Springer, Cham.

Health Europa. (2019) New chatbot therapist to help combat depression unveiled, https://www. healtheuropa.eu/chatbot-therapist-to-combat-depression-un/93609/ [Last accessed 01/02/2020]

Izquierdo LR, Olaru D, Izquierdo SS, Purchase S, Soutar GN (2015) Fuzzy logic for social simulation using NetLogo. Journal of Artificial Societies and Social Simulation 18(4):1.

Kerner C and Goodyear V A (2017) The motivational impact of wearable healthy lifestyle technologies: A self-determination perspective on FitBits with adolescents. American Journal of Health Education 48(5).

Mendel J M, John R I and Liu F (2006) Interval type-2 fuzzy logic systems made simple. IEEE Transactions on Fuzzy Systems 14(6): 808-821.

Mitleton-Kelly E (2003) Ten principles of complexity \& enabling infrastructures. In: Mitleton-Kelly E (ed) Complex Systems and Evolutionary Perspectives of Organisations: The Application of Complexity Theory to Organisations. Elsevier.

Raoufi M and Fayek A (2015). Integrating fuzzy logic and agent-based modeling for assessing construction crew behavior. In: Annual Conference of the North American Fuzzy Information Processing Society (NAFIPS) 2015 held jointly with 5th World Conference on Soft Computing (WConSC), 17-19 Aug, Redmond, Washington, USA.

Siebers P O, Aickelin U, Celia H and Clegg C W (2008) An agent-based simulation of in-store customer experiences. In: Proceedings of the OR Society Simulation Workshop (SW08), 1-2 Apr, Worcestershire, UK.

Siebers P O and Klügl F (2017) What software engineering has to offer to agent-based social simulation. In: Edmonds B and Meyer R (eds) Simulating Social Complexity - Understanding Complex Systems. Springer

Sommerville I (2015) Software Engineering. Pearson.

Vu T, Siebers P O and Wagner C (2013) Comparison of crisp systems and fuzzy systems in agentbased simulation: A case study of soccer penalties. In: Proceedings of the 2013 UK Workshop on Computational Intelligence (UKCI 2013), 9-11 Sep, Guildford, UK.

Wagner C (2013) Juzzy - A Java based toolkit for type-2 fuzzy logic. 2013 IEEE Symposium Series on Computational Intelligence, 16-19 Apr, Singapore.

Zadeh L A (1988) Fuzzy logic. Computer 21(4): 83-93

\section{AUTHOR BIOGRAPHIES}

OWEN BARNES received a MSc (with distinction) in Computer Science with Artificial Intelligence from the School of Computer Science, University of Nottingham, UK. This paper is based on his MSc dissertation.

PEER-OLAF SIEBERS is an Assistant Professor at the School of Computer Science, University of Nottingham, UK. His main research interest is the methodological advance of Computer Simulation as it is used for studying human-centric and coupled human-natural systems. He is a strong advocate of "object oriented" Agent-Based Modelling. For more see http://www.cs.nott.ac.uk/ pszps/ 